\title{
The Winfree model with non-infinitesimal phase-response curve: Ott-Antonsen theory
}

EP

Cite as: Chaos 30, 073139 (2020); https://doi.org/10.1063/5.0015131

Submitted: 25 May 2020 . Accepted: 08 July 2020. Published Online: 24 July 2020

Diego Pazó (iD), and Rafael Gallego (D)

\section{COLLECTIONS}

EP This paper was selected as an Editor's Pick
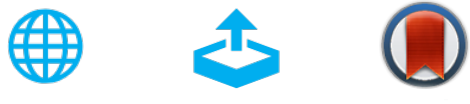

\section{ARTICLES YOU MAY BE INTERESTED IN}

Kantorovich-Rubinstein-Wasserstein distance between overlapping attractor and repeller Chaos: An Interdisciplinary Journal of Nonlinear Science 30, 073114 (2020); https:// doi.org/10.1063/5.0007230

\section{Dense networks that do not synchronize and sparse ones that do}

Chaos: An Interdisciplinary Journal of Nonlinear Science 30, 083142 (2020); https:// doi.org/10.1063/5.0018322

Dynamics and bifurcations in multistable 3-cell neural networks

Chaos: An Interdisciplinary Journal of Nonlinear Science 30, 072101 (2020); https:// doi.org/10.1063/5.0011374

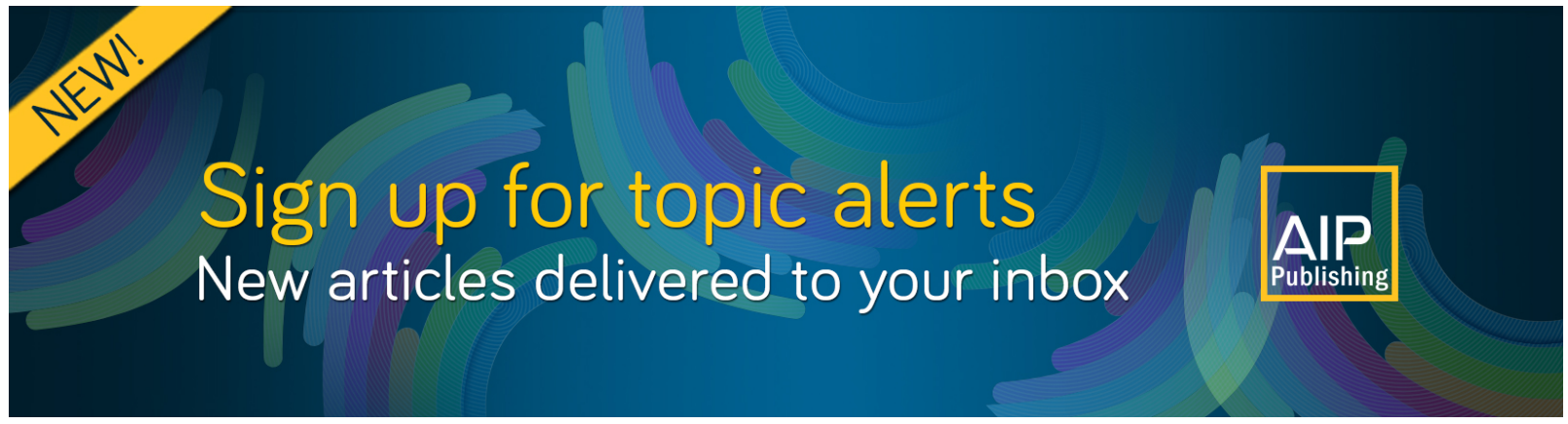




\title{
The Winfree model with non-infinitesimal phase-response curve: Ott-Antonsen theory
}

Cite as: Chaos 30, 073139 (2020); doi: $10.1063 / 5.0015131$

Submitted: 25 May 2020 . Accepted: 8 July 2020.

Published Online: 24 July 2020

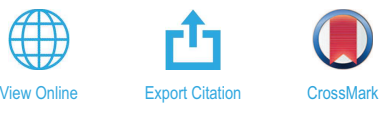

Diego Pazó ${ }^{1, a)}$ (D) and Rafael Gallego² (D)

AFFILIATIONS
${ }^{1}$ Instituto de Física de Cantabria (IFCA), CSIC-Universidad de Cantabria, 39005 Santander, Spain
${ }^{2}$ Departamento de Matemáticas, Universidad de Oviedo, Campus de Viesques, 33203 Gijón, Spain

a) Author to whom correspondence should be addressed: pazo@ifca.unican.es

\begin{abstract}
A novel generalization of the Winfree model of globally coupled phase oscillators, representing phase reduction under finite coupling, is studied analytically. We consider interactions through a non-infinitesimal (or finite) phase-response curve (PRC), in contrast to the infinitesimal PRC of the original model. For a family of non-infinitesimal PRCs, the global dynamics is captured by one complex-valued ordinary differential equation resorting to the Ott-Antonsen ansatz. The phase diagrams are thereupon obtained for four illustrative cases of non-infinitesimal PRC. Bistability between collective synchronization and full desynchronization is observed in all cases.
\end{abstract}

Published under license by AIP Publishing. https://doi.org/10.1063/5.0015131

In 1967, Winfree proposed a model for the spontaneous synchronization of large ensembles of biological oscillators. ${ }^{1}$ The Winfree model played a seminal role in the field of collective synchrony, inspiring the Kuramoto model ${ }^{2,3}$ as well as promoting recent advances in theoretical neuroscience. ${ }^{4}$ In spite of the simplifying assumptions of the Winfree model, uniform all-to-all weak coupling, analytical solutions have been found only recently using the Ott-Antonsen ansatz, ${ }^{5,6}$ see also Ref. 7 . Weak coupling is implicit in the use of phase oscillators as the units of the model. Moreover, their interactions are modeled by the so-called infinitesimal phase-response curve (iPRC), which is only valid in the limit of vanishing coupling. In this paper, we extend the Winfree model considering a non-infinitesimal (also called finite) PRC such that the phase shift of one oscillator is not proportional to the magnitude of the input. For a family of non-infinitesimal PRCs, and a Lorentzian distribution of natural frequencies, the global dynamics is captured by one complex-valued ordinary differential equation by means of the Ott-Antonsen ansatz. ${ }^{8-10}$ We obtain phase diagrams for four instructive cases.

\section{INTRODUCTION}

Collective synchronization in large ensembles of self-sustained oscillators is a pervasive phenomenon in nature and technology. ${ }^{11-13}$ The first successful attempt to model collective synchronization is due to Winfree. ${ }^{1}$ Relying on his intuition, he devised a model where the only degrees of freedom were the oscillators' phases and the coupling was uniform and global (i.e., mean-field type). In the numerical simulations, a macroscopic cluster of synchronized oscillators emerged spontaneously when either the natural frequencies of the oscillators were narrowly distributed or the coupling was large enough. In mathematical language, the phases in the Winfree model are governed by a set of $N$ ordinary differential equations $(i=1, \ldots, N)$,

$$
\begin{gathered}
\dot{\theta}_{i}=\omega_{i}+\tilde{Q}\left(\theta_{i}\right) A, \\
A=\frac{\varepsilon}{N} \sum_{j=1}^{N} P\left(\theta_{j}\right) .
\end{gathered}
$$

Here, $\omega_{i}$ is the natural frequency of the $i$ th oscillator and $\varepsilon>0$ is a parameter controlling the coupling strength. The $2 \pi$-periodic function $P$ specifies the pulse shape. Function $\tilde{Q}$ is also $2 \pi$-periodic and is either called infinitesimal (or linear) phase-response curve (iPRC) or sensitivity function. ${ }^{11,13,14}$

As already mentioned, the Winfree model relies on two assumptions: weak coupling and all-to-all geometry. First of all, weak coupling permits ignoring the oscillators' amplitudes: the limit cycles are strongly attractive compared to perturbations, causing amplitudes to be strongly damped degrees of freedom. In addition, the effect of the mean field $A$ on the phase is exactly 
proportional to $A$-higher powers of $A$ are absent in Eq. (1a) - which only holds in the limit of asymptotically small interactions. ${ }^{11,13-16}$

In this work, we generalized the Winfree model considering nonlinear interactions. Mathematical tractability imposes certain restrictions on the distribution of the natural frequencies and on the class of "non-infinitesimal" (also called "finite" or "non-linear") PRCs, but we believe it is remarkable that such analytic solutions exist. This limited progress should be welcome given the relevance of the PRC theory in theoretical neuroscience, ${ }^{17,18}$ and recent experiments evidence the insufficiency of the linear approximation. ${ }^{19,20}$ Our analysis is based on the so-called "Ott-Antonsen (OA) theory," which assumes a certain ansatz (the Poisson kernel) for the density of the phases in the thermodynamic limit $(N \rightarrow \infty)$. The OA ansatz was initially applied to the Kuramoto model and its variants $s^{8,9}$ but eventually found application in several systems of pulse-coupled oscillators: the original Winfree $\operatorname{model}^{5,6}$ (and a variant with heterogeneous iPRCs ${ }^{21}$ ), ensembles of theta neurons, ${ }^{22-24}$ quadratic integrate-and-fire neurons, ${ }^{25-27}$ and excitable active rotators. ${ }^{28,29}$

\section{WINFREE MODEL WITH NON-INFINITESIMAL PRC}

We consider a modification of the Winfree model (1), in which Eq. (1a) is replaced by

$$
\dot{\theta}_{i}=\omega_{i}+Q\left(\theta_{i}, A\right), \quad i=1, \ldots, N,
$$

where $A$ is the mean field defined by (1b). At the lowest order in $A$, model (2) converges to the Winfree model (1): $d Q\left(\theta_{i}, A\right) /\left.d A\right|_{A=0}=$ $\tilde{Q}\left(\theta_{i}\right)$. Assuming $Q(\theta, A)$ to be linear in $A$ is equivalent to approximate the isochrons of a limit cycle by straight lines (or hyperplanes if the dimensionality is larger than two) in the phase reduction procedure. $^{13,14}$

\section{A. Non-infinitesimal PRC}

Prior to specifying the PRC Q, we devote a few lines to iPRCs. Traditionally, iPRCs are classified as type I or type II. ${ }^{30}$ For type II, either an advance or a delay in the phase are possible depending upon the timing of the perturbation, while in the case of type I, the timing of the perturbation does not change the sign of the phase shift. The canonical examples of each type ${ }^{13,15}$ are $\tilde{Q}(\theta) \propto 1-\cos \theta$ for type I (e.g., the theta neuron) and $\widetilde{Q}(\theta) \propto \sin \theta$ for type II (e.g., the Stuart-Landau oscillator). For non-infinitesimal PRCs, the previous classification falls short as the character of $Q$ may change with the strength of the stimulus. ${ }^{13,15}$

The types of PRC we consider are conditioned by the applicability of the OA ansatz, as it enables a drastic dimensionality reduction. The OA ansatz imposes that no harmonics in $\theta$ beyond the first one are present in $Q(\theta, A)$. Still, the family of PRCs with only first harmonic in $\theta$ is wide enough to make the problem nontrivial. As we shall adopt pulses $P(\theta)$ with peak value at $\theta=0$ (and multiples of $2 \pi$ ), we impose the additional constraint $Q(0, A)=0$ motivated by the fact that the PRC vanishes at spiking/flashing times for most neurons $\mathrm{s}^{31,32}$ and certain fireflies. ${ }^{33,34}$ Therefore, we restrict to a family of PRCs of this form,

$$
Q(\theta, A)=f_{1}(A)(1-\cos \theta)-f_{2}(A) \sin \theta,
$$

where $f_{1}$ and $f_{2}$ are arbitrary functions of $A$, provided $f_{1,2}(0)=0$ for obvious physical reasons. In similarity with the classification of

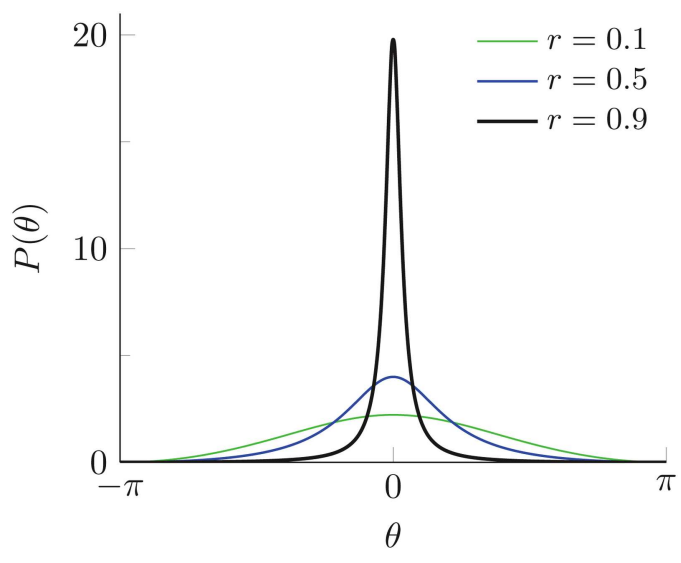

FIG. 1. Rectified-Poisson pulse (4) for three values of parameter $r$.

iPRCs, we refer to the two terms in (3), proportional to $(1-\cos \theta)$ and $\sin \theta$, as the type I and the type II components of the PRC, respectively.

\section{B. Pulse shape}

In the study of the classical Winfree model, several pulse shapes can be considered, see Ref. 6. In this work, we adopt a "rectifiedPoisson kernel",

$$
P(\theta)=\frac{(1-r)(1+\cos \theta)}{1-2 r \cos \theta+r^{2}} .
$$

This is a particularly convenient shape for the theoretical analysis below. $P(\theta)$ is a symmetric unimodal function in the interval $\left[-\pi, \pi\right.$ ] [with the normalization $\int_{-\pi}^{\pi} P(\theta) d \theta=2 \pi$ ] that peaks at $\theta=0$ and vanishes at $\theta= \pm \pi$. Parameter $r$ is a real number allowing a continuous interpolation between a flat pulse for $r=-1$ and a Dirac-delta pulse, $P(\theta)=2 \pi \delta(\theta)$, for $r=1$. In Fig. 1, the pulse function $P(\theta)$ is depicted for three different values of $r$.

\section{Natural frequencies}

For the sake of achieving the maximal dimensionality reduction, we assume the natural frequencies to be distributed according to a Lorentzian distribution of half-width $\Delta$ centered at $\omega_{0}$,

$$
g(\omega)=\frac{\Delta / \pi}{\left(\omega-\omega_{0}\right)^{2}+\Delta^{2}} .
$$

\section{OTT-ANTONSEN THEORY}

Once the building blocks of the model have been introduced, we apply the OA theory. ${ }^{8}$ In this way, we derive a complex-valued ODE reproducing the long-time evolution of the model at the macroscopic level. As the procedure is standard, ${ }^{6,8}$ the readers interested in the final result are pointed to Eqs. (10) and (11).

First of all, one must realize that our model (2) belongs to a general class of oscillator systems of the form

$$
\dot{\theta}_{i}(t)=\omega_{i}+B(t)+\operatorname{Im}\left[H(t) e^{-i \theta_{i}(t)}\right],
$$


which can be analyzed with the OA ansatz. ${ }^{8-10,35}$ Functions $B$ and $H$ may depend explicitly on time or indirectly through a mean field. For PRC (3), we have

$$
B(t)=f_{1}(A), \quad H(t)=f_{2}(A)-i f_{1}(A) .
$$

In the thermodynamic limit, we can define a phase density $F(\theta \mid \omega, t)$ such that $F(\theta \mid \omega, t) d \theta$ is the fraction of oscillators of frequency $\omega$ at time $t$, with phases in the interval $[\theta, \theta+d \theta]$. It is convenient to introduce the Fourier expansion of the density,

$$
F(\theta \mid \omega, t)=\sum_{m=-\infty}^{\infty} \alpha_{m}(\omega, t) e^{i m \theta},
$$

with $\alpha_{-m}=\alpha_{m}^{*}$. We notice as well that, by conservation of the number of oscillators, $F$ satisfies the continuity equation: $\partial_{t} F+\partial_{\theta}(F \dot{\theta})=$ 0 , where $\theta$ is the speed of an oscillator of natural frequency $\omega$. Inserting the Fourier series of $F$ into the continuity equation, we get

$$
\partial_{t} \alpha_{m}(\omega, t)=-i m(\omega+B) \alpha_{m}+\frac{m}{2}\left(H^{*} \alpha_{m-1}-H \alpha_{m+1}\right) .
$$

A particular solution of this equation, the OA ansatz, is obtained equating the coefficient of $m$ th mode to the $m$ th power of the first mode: $\alpha_{m}=\alpha_{1}^{m}$. Hence, for the solution in this so-called OA manifold, we only need to consider the evolution of $\alpha_{1} \equiv \alpha$,

$$
\partial_{t} \alpha(\omega, t)=-i(\omega+B) \alpha+\frac{1}{2}\left(H^{*}-H \alpha^{2}\right) .
$$

This is still an infinite set of coupled integrodifferential equations. A sharp reduction in the dimensionality of the problem is achieved for rational $g(\omega)$ and specially for the Lorentzian distribution. ${ }^{8}$ As the Kuramoto order parameter ${ }^{2} Z=\overline{e^{i \theta}}$ is related to $\alpha$ via $Z^{*}(t)=$ $\int_{-\infty}^{\infty} \alpha(\omega, t) g(\omega) d \omega$, we can evaluate this integral resorting to the residue theorem obtaining $Z^{*}(t)=\alpha\left(\omega_{0}-i \Delta, t\right)$. (This is the result of performing an analytic continuation of $\alpha$ from real to complex $\omega$ and evaluating $\alpha$ at the pole of $g(\omega)$ in the lower half $\omega$-plane.) Thus, setting $\omega=\omega_{0}-i \Delta$ in (9), we get a complex-valued ODE for the Kuramoto order parameter,

$$
\dot{Z}=\left(-\Delta+i \omega_{0}\right) Z-\frac{i}{2} f_{1}(A)(1-Z)^{2}+\frac{1}{2} f_{2}(A)\left(1-Z^{2}\right),
$$

where $B$ and $H$ have been written in terms of $f_{1}$ and $f_{2}$ according to Eq. (7). To close Eq. (10), we need to express the mean field $A$ as a function of $Z$. For the pulse shape in Eq. (4) and a Lorentzian frequency distribution, it can be proven (see Ref. 6 or the supplementary material of Ref. 4 ) that

$$
A=\varepsilon \operatorname{Re}\left(\frac{1+Z}{1-r Z}\right) \text {. }
$$

Note that $0 \leq A \leq A_{\max }$, where the maximal value $A_{\max }=2 \varepsilon /(1-$ $r$ ) is achieved if $Z=1$ (all oscillators exactly at $\theta_{j}=0$ ). In addition to this, the central natural frequency $\omega_{0}$ is hereafter set to 1 , as this can always be achieved by rescaling time and $f_{1,2}$.

\section{FOUR ILLUSTRATIVE PRCs}

Among the infinite set of functions $f_{1}(A)$ and $f_{2}(A)$, we selected a few illustrative case studies. In each of these cases, the character of the PRC undergoes a crossover as $A$ grows: from one iPRC
TABLE I. The four cases of non-infinitesimal PRCs analyzed in this paper. Functions $f_{1}$ and $f_{2}$ determine the PRC in $(3)[\sigma(A)$ is a crossover function, see (12)]. The code in the last column indicates the PRC and the asymptotic PRC at large $A$ (see text).

\begin{tabular}{lccc}
\hline \hline Case & $f_{1}(A)$ & $f_{2}(A)$ & Code \\
\hline $\mathrm{a}$ & $\sigma(A)$ & $A \sigma(A)$ & $\mathrm{I}-\mathrm{II}_{s}$ \\
$\mathrm{~b}$ & $A \sigma(A)$ & $\sigma(A)$ & $\mathrm{II}_{s}-\mathrm{I}$ \\
$\mathrm{c}$ & 0 & $(1-A) \sigma(A)$ & $\mathrm{II}_{s}-\mathrm{II}_{r}$ \\
$\mathrm{~d}$ & 0 & $-(1-A) \sigma(A)$ & $\mathrm{II}_{r}-\mathrm{II}_{s}$ \\
\hline \hline
\end{tabular}

type to a different PRC type for large $A$. We denote the limiting PRC at $A \rightarrow \infty$ as "asymptotic PRC" (aPRC). From now on, we apply the classical distinction between types I and II to both iPRCs and aPRCs. Recall that if the sign is the same for all $\theta$, we call the iPRC (or the aPRC) as type I (implying $f_{2}=0$ ), while in the complementary case with $f_{1}=0$, we refer to the iPRC (or to the aPRC) as canonical type II or simply type II. Notably, type II may either promote or impede synchronization depending on the sign of $f_{2}$. In turn, we distinguish between two subclasses of type II: $\mathrm{II}_{s}\left(f_{2}>0\right)$ and $\mathrm{II}_{r}\left(f_{2}<0\right)$ corresponding to the synchronizing and repulsive interactions, respectively.

As we are interested in introducing one crossover in the PRC between the iPRC and the aPRC and have three fundamentally different types $\left(\mathrm{I}, \mathrm{II}_{s}\right.$, and $\left.\mathrm{II}_{r}\right)$, this gives six possible combinations. However, we shall consider only four of these iPRC-aPRC pairs, since only type $\mathrm{II}_{s}$ favors synchrony and is to be included either in the iPRC or in the aPRC. Otherwise, no synchronization phenomena are expected: type I is neutral and type $\mathrm{II}_{r}$ is repulsive. Hence, we focus on the four cases listed in Table I, in which different PRC types characterize small and large $A$ regimes. As a guide, in the fourth column of the table, we write a code $\mathrm{X}-\mathrm{Y}$, where $\mathrm{X}$ refers to the iPRC and $\mathrm{Y}$ to the aPRC. The saturation function $\sigma(A)$ in the table has positive slope at $A=0$ and saturates at large $A$. In particular, we chose this specific saturation function in our study,

$$
\sigma(A)=\frac{A}{1+A} .
$$

(Our results have been occasionally tested against another choice $\sigma(A)=\tanh (A)$, finding no qualitative difference.) Graphical representations of the four PRCs (cases a-d), for four representative $A$ values, are shown in Figs. 2(a)-2(d). In each panel, the PRC appears divided by $A$ as usual, ${ }^{13}$ and the lack of overlapping between different lines evidences its nonlinearity.

In Sec. $V$, we obtain the phase diagrams corresponding to each of the four cases introduced here, based on the analysis of the complex-valued ordinary differential Eq. (10). But before doing so, it is worth making direct simulations of full system (2) and test (and understand) the correspondence with the solutions of Eq. (10). We simulated the full model in case $\mathrm{d}$ with $\omega_{0}=1$, heterogeneity parameter $\Delta=0.01$, pulse-shape parameter $r=0.9$, and coupling constant $\varepsilon=0.4$. As may be seen in Fig. 3, the population exhibits bistability between a desynchronized state and a synchronized state with some oscillators oscillating with the same frequency. This bistability is not surprising as the system is "more synchronizing" when already synchronized since the aPRC is of type $\mathrm{II}_{s}$, while it is hardly 

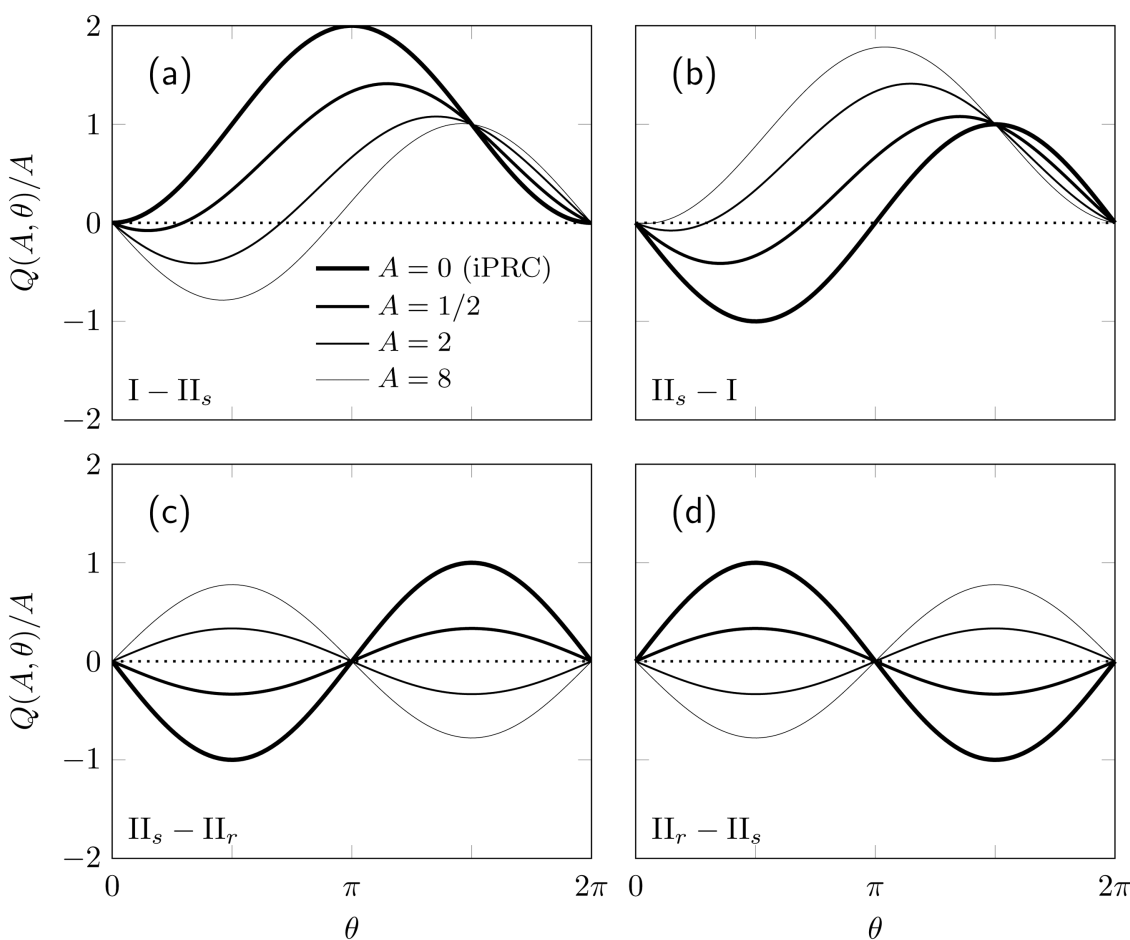

FIG. 2. The non-infinitesimal PRCs analyzed in this work as a function of $\theta$ for four representative values of $A$, including $A=0^{+}$(iPRC) and $A=8$ (resembling the aPRC). Panels (a)-(d) correspond to cases a-d, respectively (see Table I). The code IPRC-aPRC is indicated in each panel. synchronizable when already desynchronized by virtue of the type $\mathrm{II}_{r}$ iPRC. In terms of $Z$, the synchronous solution is (approximately) a periodic orbit, while the desynchronized state exhibits only small fluctuations around a point due to finite size effects $(N=500)$. The agreement with the stable fixed point and the stable limit cycle of Eq. (10), also represented in Figs. 3(b) and 3(d), is excellent.

\section{PHASE DIAGRAMS}

In the remainder of this paper, we obtain the phase diagrams for the four reference cases by means of Eq. (10). As (10) is a (generic) planar system, the only possible attractors are fixed points and limit cycles. Their bifurcation loci, depicted in the phase diagrams below, have been obtained using the MATCONT toolbox ${ }^{36}$ of MATLAB. Moreover, we recall that $Z$ is only physically meaningful inside the unit disk $|Z| \leq 1$ and, therefore, attractors and bifurcations occurring outside it are ignored. As seen in Fig. 3, limit cycles correspond to synchronized solutions in which a macroscopic part of the population rotates at the same average frequency.

\section{A. Case a: I- II s}

In Fig. 4(a), we show the phase diagram spanned by parameters $\Delta$ and $\varepsilon$. Bifurcation lines for three values of parameter $r$, controlling the pulse width, are depicted. The results almost replicate those in Ref. 6 for the standard Winfree model with type $\mathrm{II}_{s}$ iPRC. Synchronization is found in two adjacent regions, in one of them (dark shaded) coexisting with a desynchronized state. [There exists a region (not shown) besides the bistability region where two desynchronized states coexist, see Refs. 5 and 6]. In contrast to the averaging approximation (the Kuramoto-Sakaguchi model), valid at small $\varepsilon$ and $\Delta$, synchronization becomes impossible if the population is too heterogeneous (large $\Delta$ ).

For small coupling (and heterogeneity), synchronization emerges from a supercritical Hopf bifurcation undergone by the desynchronized state, akin to the classical Kuramoto transition. ${ }^{8}$ This Hopf bifurcation line terminates at a double zero eigenvalue (Bogdanov-Takens, BT) point. A homoclinic (Hom) line emanates from the BT point limiting the coexistence region. As observed for the regular Winfree model, ${ }^{5,6}$ synchronization is more efficient for narrow pulses. The pulse width does not qualitatively change the phase diagram.

The phase diagram only differs appreciably from those in Ref. 6 at the origin. We see that, due to the type I iPRC, the Hopf line approaches the origin with an infinite slope. In particular, the asymptotic dependence of the critical $\varepsilon_{H}$ on $\Delta$ follows an unusual square-root law with the frequency dispersion $\Delta$,

$$
\varepsilon_{H}=\sqrt{\frac{2 \Delta}{1+r}} .
$$

We can deduce this result deriving the associated KuramotoSakaguchi model of model (2) via averaging. Or, alternatively, preserving in (10) only linear, rotationally invariant terms in $Z$ and equating the linear coefficient to $i \Omega$.

\section{B. Case b: $I I_{s}-\mathrm{I}$}

In case $b, i P R C$ and aPRC are interchanged with respect to case a. This means that synchronization is favored at small coupling but becomes increasingly difficult as the coupling grows. Accordingly, 

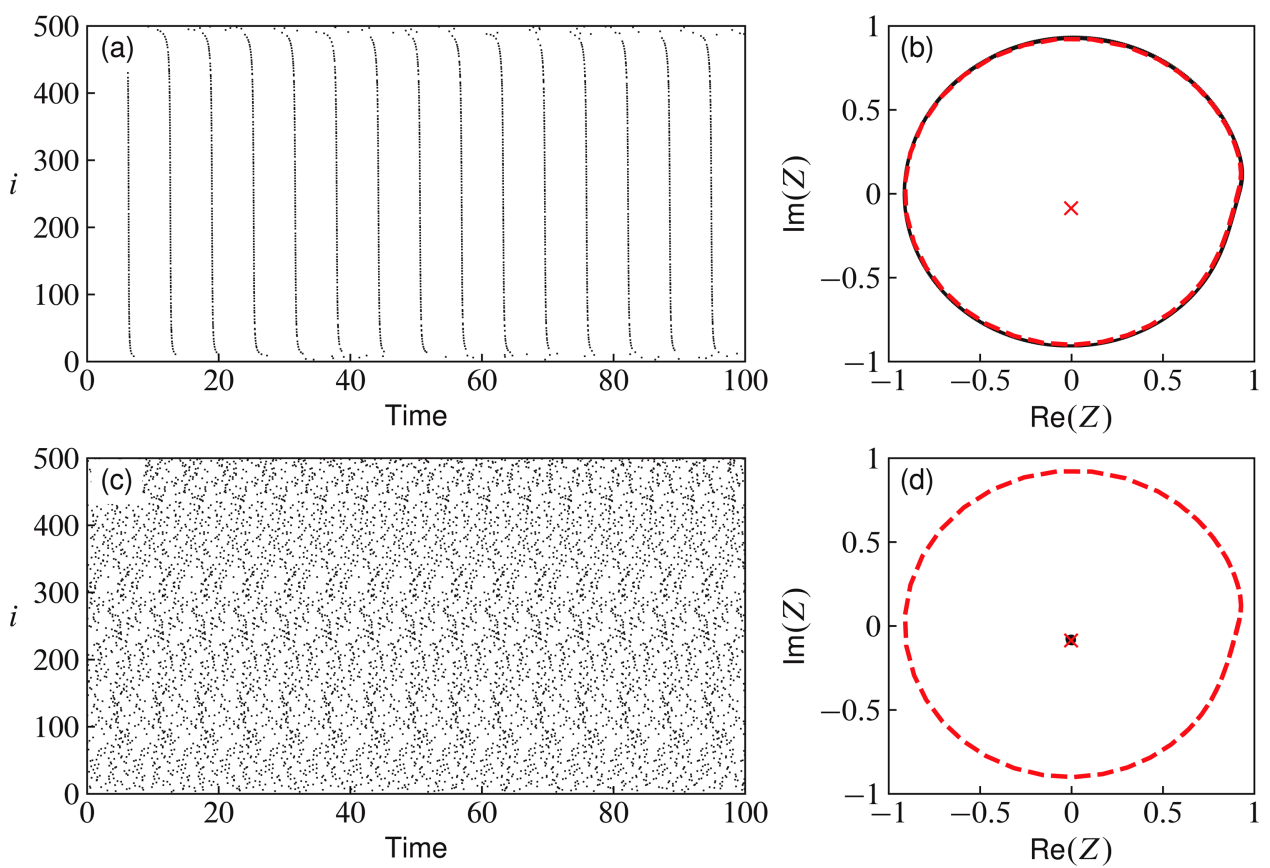

FIG. 3. (a) Raster plot-a dot indicates at which time one oscillator phase crosses a multiple of $2 \pi-$ for a population of $N=500$ and the non-infinitesimal PRC of case $d$, see Table I and Fig. 2(d). The initial condition is uniform $\theta_{j}(t=0)=0.01$, and parameters are $\Delta=0.01, r=0.9, \varepsilon=0.4$. The frequencies are deterministically drawn from a Lorentzian distribution: $\omega_{i}=\omega_{0}+\Delta \tan [\pi(2 i-N-1) /(2 N)]$. (c) The same as (a) but for random initial distribution of phases. (b) and (d) depict the Kuramoto order parameter $Z(t)=N^{-1} \sum_{j} e^{i \theta_{j}(t)}$ for 50 t.u., once the simulations in (a) and (c), respectively, reached the stationary state. The red dashed line and the red cross in panels (b) and (d) are the periodic and fixed point attractors of Eq. (10), coexisting at the same parameter values.

the phase diagram in Fig. 4(b) shows the expected supercritical Hopf bifurcation line emanating as a straight line from the origin: ${ }^{5,6} \varepsilon_{H} \propto$ $\Delta+O\left(\Delta^{2}\right)$.

At large $\varepsilon$, there is a bistability region such that the synchronized state disappears in a saddle-node bifurcation of limit cycles (SNLCs). The locus of the SNLC is a line that emanates from a generalized Hopf (or Bautin) point (GH) and terminates at the $\varepsilon$ axis at a point marked with a star on the $\varepsilon$-axis of the phase diagram. The stars pinpoint the (equivariant) transcritical (TC) bifurcation, ${ }^{37}$ in which the fully synchronized state $\left[\theta_{i}(t)=\theta_{j}(t)\right]$ of identical oscillators $(\Delta=0)$ becomes unstable. For $r=0.9$, the instability of full synchronization takes place at $\varepsilon_{c}=9.555 \ldots$, far above the range of $\varepsilon$ displayed in the phase diagram. The location of $\varepsilon_{c}$ was not calculated using (10) but by directly looking for the stability threshold to the fully synchronized state, see the Appendix.

Finally, note that the synchronization region shrinks as the pulse becomes wider, but there is not a qualitative change in the phase diagram whatsoever.

\section{Case c: $\mathrm{II}_{s}-\mathrm{II}_{r}$}

In this case, the aPRC is repulsive, in contrast to case b, where the aPRC is type I (i.e., neutral in terms of synchronization). In turn, the phase diagram in Fig. 4(c) shows a quite small synchronization region (notice the scale of the axes). Synchronization is bounded exclusively by a supercritical Hopf bifurcation, save for broad pulses.
In the latter case, a GH point is found, and the Hopf bifurcation is subcritical at the left of it. Accordingly, we find a bistability region bounded by a line of saddle-node bifurcation of limit cycles (SNLCs) and a subcritical Hopf bifurcation, as in case $b$. The precise value of $r$ below which the bistability region exists (i.e., the $\mathrm{GH}$ point is present) is $r_{*} \simeq 0.27891$.

Note also the presence of a TC point in the phase diagram at $\Delta=0$, above which full synchrony destabilizes. ${ }^{38}$ The transcritical bifurcation is not structurally stable, see, e.g., Fig. 1 in Ref. 39 and increasing $\Delta$ from 0 may either leave no trace of bifurcation or "decay" into two saddle-node bifurcation of limit cycles. The latter scenario occurs for $r<0.27577 \ldots$, see the bifurcation lines for $r=0.1$ in Fig. 4(c), but in our case, one of the bifurcations is not shown as it entails $|Z|>1$.

\section{Case d: $\mathrm{II}_{r}-\mathrm{II}_{s}$}

Case $\mathrm{d}$ exhibits the most complex phase diagram among all those obtained here. The aPRC is of type $\mathrm{II}_{s}$, as in case a, and (accordingly) the large $\epsilon$ region is organized by two codimensiontwo points: the Bogdanov-Takens (BT) and the saddle-node separatrix-loop (SNSL) codimension-two points. The associated region of bistability between synchrony and asynchrony is bounded by homoclinic, saddle-node, and Hopf bifurcations.

Remarkably, there is also a bistability region at small $\varepsilon$ values for $r>r_{*} \simeq 0.27891$ (recall the simulations in Fig. 3), which 

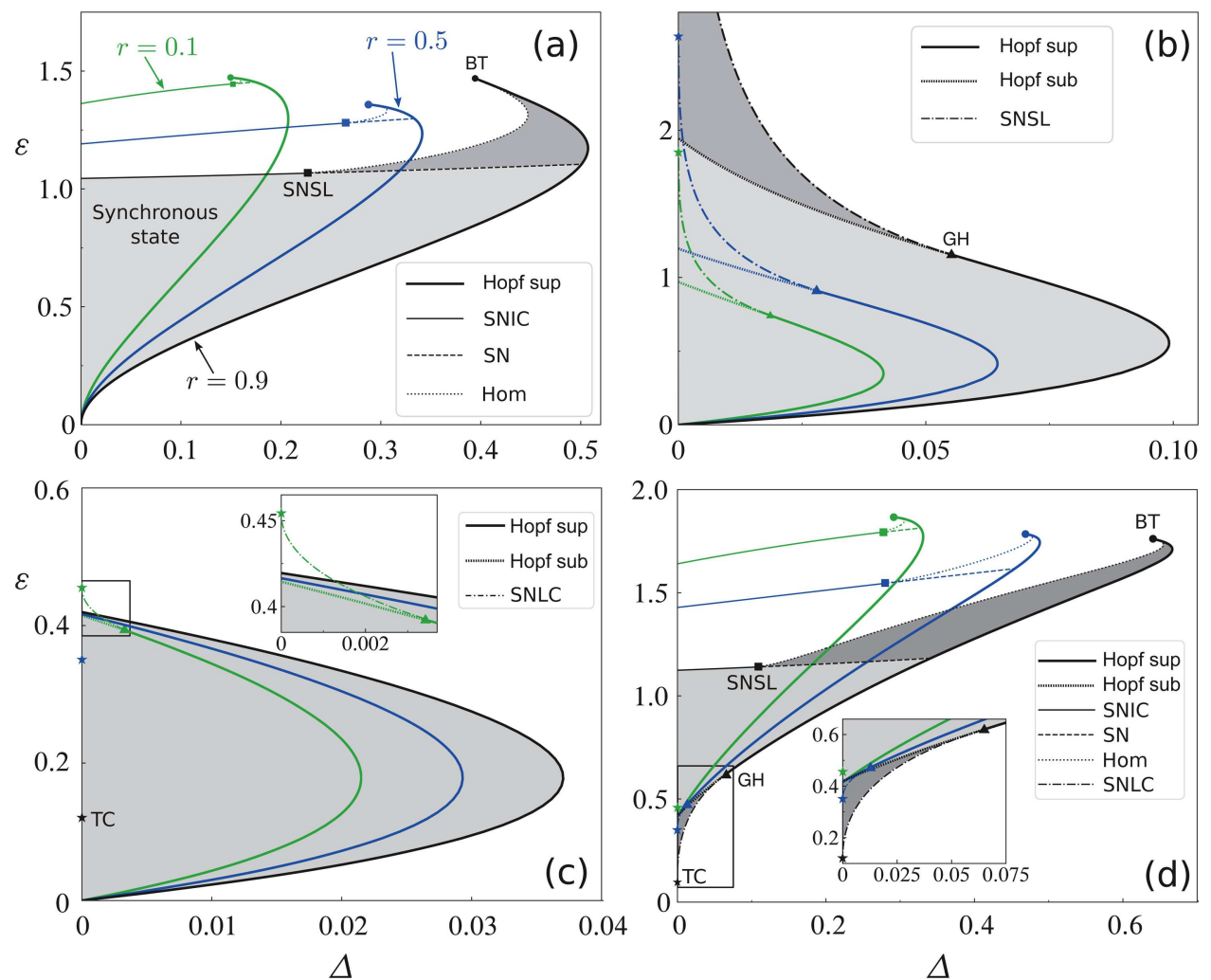

FIG. 4. Synchronization regions of the Winfree model in the $(\Delta, \varepsilon)$-plane for the four cases of PRCs described in Table I, and three different values of the parameter $r \in\{0.1,0.5,0.9\}$. Panels (a)-(d) correspond to cases a-d, respectively. For the value $r=0.9$, light shaded regions indicate where there is a stable limit cycle, corresponding to a macroscopic synchronized state. In the dark shaded regions, the limit cycle (synchronous state) coexists with a stable fixed point (asynchronous state). The

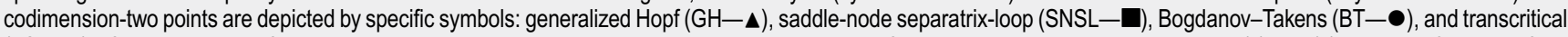
(TC- $\star$ ) bifurcations. The bifurcation corresponding to each line type is indicated in the legend of the respective panel. Insets in panels (c) and (d) are magnifications of the regions inside the respective rectangles.

is bounded by a subcritical Hopf and a saddle-node of limit cycles bifurcations. In contrast to previous cases, this synchronization region is detached from the origin due to the repulsive character of the iPRC. To be more precise, the bottom corner of the lower bistability region located at point TC approaches the origin as $r \rightarrow 1$.

\section{CONCLUSIONS}

In this work, we have studied a non-trivial extension of the Winfree model in which the PRC is nonlinear in the mean field. If the PRC contains only the first harmonic of the angle, the OA ansatz permits a sharp dimensionality reduction. Among all possible dependencies of the PRC on the mean field, we have considered only those with a crossover between two different canonical components. In particular, we have analyzed four cases in which an attractive type II component competes either against a repulsive type II component or against a type I component. Synchronization regions are peculiar for each case. Bistability between macroscopic synchronization and complete desynchronization is found in all cases (in case c, only for broad pulses), but in different relative locations in the $\Delta-\varepsilon$ plane.

Our results indicate that the nonlinearity of the PRC with the forcing, by itself, is not enough to generate complex collective phenomena. This is certain for a Lorentzian distribution of frequencies since the reduced system is only two dimensional, irrespective of the exact form of $f_{1}(A)$ and $f_{2}(A)$. As happens in Kuramotolike models, phenomena such as clustering or glassy dynamics may require multiple Fourier components ${ }^{40}$ (in the PRC) or stronger heterogeneity, ${ }^{41}$ respectively. Concerning collective chaos, other ingredients such as a time-varying coupling, ${ }^{42}$ two interacting populations, ${ }^{43}$ or multimodal frequency distributions ${ }^{44}$ appear to be imperative.

Needless to say, our study is only a drop in the ocean of possible PRCs and model generalizations. For instance, relaxation oscillators $^{45}$ and bursting (neuronal) oscillators ${ }^{46}$ have PRCs very different from the first-harmonic shape function in Eq. (3). Nevertheless, in spite of its limitations, we regard the model defined by Eqs. (2) and (3) as a noteworthy example of a system in which the OA theory can be fully applied. 


\section{ACKNOWLEDGMENTS}

We acknowledge support by the Agencia Estatal de Investigación and Fondo Europeo de Desarrollo Regional under Project No. FIS2016-74957-P (AEI/FEDER, EU).

\section{APPENDIX: IDENTICAL OSCILLATORS}

If the oscillators are identical, there is a fully synchronized solution $\theta_{j}(t)=\Psi(t)$. The dynamics of $\Psi$ obeys

$$
\dot{\Psi}=\omega_{0}+f_{1}[\varepsilon P(\Psi)](1-\cos \Psi)-f_{2}[\varepsilon P(\Psi)] \sin \Psi .
$$

Next, we calculate the stability threshold of full synchrony, fixing $\omega_{0}=1$ as in the main text. In the thermodynamic limit $(N \rightarrow \infty)$, we may perturb one oscillator, say, the first one, without changing the mean field. Hence, one infinitesimal perturbation $\delta \theta=\theta_{1}-\Psi$ obeys

$$
\dot{\delta \theta}=\lambda(\Psi) \delta \theta,
$$

where the multiplicative factor $\lambda(\Psi)=f_{1}[\varepsilon P(\Psi)] \sin \Psi-f_{2}[\varepsilon P(\Psi)]$ $\cos \Psi$ depends on time through $\Psi(t)$. In order to know the average exponential growth (or contraction) rate of $\delta \theta$, we need to integrate over variable $\Psi$, taking into account its density $\rho(\Psi)$. These mean that the sign of constant $\lambda$, given by

$$
\lambda=\int_{-\pi}^{\pi} \lambda(\Psi) \rho(\Psi) d \Psi,
$$

determines the stability of the fully synchronized solution. If $\lambda$ is positive, the oscillator "evaporates" from the main cluster, i.e., full synchrony is unstable.

The density $\rho(\Psi)$ is proportional to the inverse of the speed: $\rho(\Psi) \propto \dot{\Psi}^{-1}$. Imposing $\lambda=0$, we obtain the condition for the stability threshold of full synchrony,

$$
\int_{-\pi}^{\pi} \frac{f_{1}\left[\varepsilon_{c} P(\Psi)\right] \sin \Psi-f_{2}\left[\varepsilon_{c} P(\Psi)\right] \cos \Psi}{1+f_{1}\left[\varepsilon_{c} P(\Psi)\right](1-\cos \Psi)-f_{2}\left[\varepsilon_{c} P(\Psi)\right] \sin \Psi} d \Psi=0 .
$$

This integral cannot be solved analytically, but the threshold coupling $\varepsilon_{c}$ is easily found numerically.

\section{DATA AVAILABILITY}

The data that support the findings of this study are available from the corresponding author upon reasonable request.

\section{REFERENCES}

${ }^{1}$ A. T. Winfree, "Biological rhythms and the behavior of populations of coupled oscillators," J. Theor. Biol. 16, 15-42 (1967).

${ }^{2}$ Y. Kuramoto, "Self-entrainment of a population of coupled non-linear oscillators," in International Symposium on Mathematical Problems in Theoretical Physics, Lecture Notes in Physics Vol. 39, edited by H. Araki (Springer, Berlin, 1975), pp. 420-422.

${ }^{3}$ S. H. Strogatz, "From Kuramoto to Crawford: Exploring the onset of synchronization in populations of coupled oscillators," Physica D 143, 1-20 (2000).

${ }^{4}$ E. Montbrió and D. Pazó, "Kuramoto model for excitation-inhibition-based oscillations,” Phys. Rev. Lett. 120, 244101 (2018).

${ }^{5}$ D. Pazó and E. Montbrió, "Low-dimensional dynamics of populations of pulsecoupled oscillators,” Phys. Rev. X 4, 011009 (2014).

${ }^{6}$ R. Gallego, E. Montbrió, and D. Pazó, "Synchronization scenarios in the Winfree model of coupled oscillators,” Phys. Rev. E 96, 042208 (2017).
${ }^{7}$ J. T. Ariaratnam and S. H. Strogatz, "Phase diagram for the Winfree model of coupled nonlinear oscillators," Phys. Rev. Lett. 86, 4278-4281 (2001).

${ }^{8}$ E. Ott and T. M. Antonsen, "Low dimensional behavior of large systems of globally coupled oscillators," Chaos 18, 037113 (2008)

${ }^{9}$ E. Ott and T. M. Antonsen, "Long time evolution of phase oscillator systems," Chaos 19, 023117 (2009).

${ }^{10}$ E. Ott, B. R. Hunt, and T. M. Antonsen, "Comment on 'Long time evolution of phase oscillators systems,'” Chaos 21, 025112 (2011).

${ }^{11}$ A. T. Winfree, The Geometry of Biological Time (Springer, New York, 1980).

${ }^{12}$ A. S. Pikovsky, M. G. Rosenblum, and J. Kurths, Synchronization: A Universal Concept in Nonlinear Sciences (Cambridge University Press, Cambridge, 2001).

${ }^{13}$ E. M. Izhikevich, Dynamical Systems in Neuroscience (The MIT Press, Cambridge, MA, 2007), Chap. 10.

${ }^{14}$ Y. Kuramoto, Chemical Oscillations, Waves, and Turbulence (Springer-Verlag, Berlin, 1984).

${ }^{15} \mathrm{P}$. Sacré and R. Sepulchre, "Sensitivity analysis of oscillator models in the space of phase-response curves: Oscillators as open systems," IEEE Control Syst. Mag. 34, 50-74 (2014).

${ }^{16}$ B. Pietras and A. Daffertshofer, "Network dynamics of coupled oscillators and phase reduction techniques," Phys. Rep. 819, 1-105 (2019).

${ }^{17}$ G. B. Ermentrout and D. H. Terman, Mathematical Foundations of Neuroscience (Springer, 2010), Vol. 64

${ }^{18} \mathrm{C}$. Börgers, An Introduction to Modeling Neuronal Dynamics, Texts in Applied Mathematics (Springer International Publishing, 2017).

${ }^{19}$ J. Rode, J. F. Totz, E. Fengler, and H. Engel, "Chimera states on a ring of strongly coupled relaxation oscillators,” Front. Appl. Math. Stat. 5, 31 (2019).

${ }^{20}$ D. Călugăru, J. F. Totz, E. A. Martens, and H. Engel, "First-order synchronization transition in a large population of relaxation oscillators," arXiv:1812.04727.

${ }^{21}$ D. Pazó, E. Montbrió, and R. Gallego, "The Winfree model with heterogeneous phase-response curves: Analytical results,” J. Phys. A Math. Theor. 52, 154001 (2019).

${ }^{22}$ T. B. Luke, E. Barreto, and P. So, "Complete classification of the macroscopic behavior of a heterogeneous network of theta neurons," Neural Comput. 25, 3207-3234 (2013)

${ }^{23}$ C. R. Laing, "Derivation of a neural field model from a network of theta neurons," Phys. Rev. E 90, 010901 (2014).

${ }^{24}$ P. So, T. B. Luke, and E. Barreto, "Networks of theta neurons with timevarying excitability: Macroscopic chaos, multistability, and final-state uncertainty," Physica D 267, 16-26 (2014).

${ }^{25}$ E. Montbrió, D. Pazó, and A. Roxin, "Macroscopic description for networks of spiking neurons," Phys. Rev. X 5, 021028 (2015).

${ }^{26} \mathrm{D}$. Pazó and E. Montbrió, "From quasiperiodic partial synchronization to collective chaos in populations of inhibitory neurons with delay," Phys. Rev. Lett. 116, 238101 (2016).

${ }^{27}$ I. Ratas and K. Pyragas, "Macroscopic self-oscillations and aging transition in a network of synaptically coupled quadratic integrate-and-fire neurons," Phys. Rev. E 94, 032215 (2016).

${ }^{28}$ K. P. O'Keeffe and S. H. Strogatz, "Dynamics of a population of oscillatory and excitable elements,” Phys. Rev. E 93, 062203 (2016).

${ }^{29}$ J. Roulet and G. B. Mindlin, "Average activity of excitatory and inhibitory neural populations,” Chaos 26, 093104 (2016).

${ }^{30}$ D. Hansel, G. Mato, and C. Meunier, "Synchrony in excitatory neural networks," Neural Comput. 7, 307-337 (1995).

${ }^{31}$ A. D. Reyes and E. E. Fetz, "Two modes of interspike interval shortening by brief transient depolarizations in cat neocortical neurons," J. Neurophysiol. 69, 1661-1672 (1993).

${ }^{32}$ T. I. Netoff, M. I. Banks, A. D. Dorval, C. D. Acker, J. S. Haas, N. Kopell, and J. A. White, "Synchronization in hybrid neuronal networks of the hippocampal formation,” J. Neurophysiol. 93, 1197-1208 (2005).

${ }^{33} \mathrm{~J}$. Buck, "Synchronous rhythmic flashing of fireflies II," Q. Rev. Biol. 63, 265-289 (1988).

${ }^{34}$ F. E. Hanson, "Comparative studies of firefly pacemakers," Fed. Proc. 37, 2158-2164 (1978).

${ }^{35}$ B. Pietras and A. Daffertshofer, "Ott-Antonsen attractiveness for parameterdependent oscillatory systems,” Chaos 26, 103101 (2016). 
${ }^{36}$ A. Dhooge, W. Govaerts, and Y. A. Kuznetsov, "MATCONT: A matlab package for numerical bifurcation analysis of ODEs," SIGSAM Bull. 38, 21-22 (2004).

${ }^{37} \mathrm{P}$. Ashwin and J. W. Swift, "The dynamics of $\mathrm{n}$ weakly coupled identical oscillators," J. Nonlin. Sci. 2, 69-108 (1992).

${ }^{38}$ As the "OA manifold" is not attracting for identical oscillators, ${ }^{8-10}$ the resulting dynamics depends on the initial conditions. [For initial conditions in the OA manifold, such as purely random phases $(Z=0)$, the system converges to a state of quasiperiodic partial synchronization, ${ }^{47,48}$ a state at which $Z$ oscillates periodically, while the individual oscillators behave quasiperiodically.]

${ }^{39}$ J. D. Crawford, "Introduction to bifurcation theory," Rev. Mod. Phys. 63, 991-1037 (1991).

${ }^{40} \mathrm{~K}$. Okuda, "Variety and generality of clustering in globally coupled oscillators," Physica D 63, 424-436 (1993).

${ }^{41}$ D. Iatsenko, P. V. E. McClintock, and A. Stefanovska, "Oscillator glass in the generalized Kuramoto model: Synchronous disorder and two-step relaxation," Nat. Commun. 5, 4188 (2014).
${ }^{42} \mathrm{P}$. So and E. Barreto, "Generating macroscopic chaos in a network of globally coupled phase oscillators," Chaos 21, 033127 (2011).

${ }^{43}$ C. Bick, M. J. Panaggio, and E. A. Martens, "Chaos in Kuramoto oscillator networks," Chaos 28, 071102 (2018)

${ }^{44}$ H. Cheng, S. Guo, Q. Dai, H. Li, and J. Yang, "Collective chaos and perioddoubling bifurcation in globally coupled phase oscillators," Nonlinear Dyn. 89, 2273-2281 (2017)

${ }^{45} \mathrm{P}$. Sacré and A. Franci, "Singularly perturbed phase response curves for relaxation oscillators," in 2016 IEEE 55th Conference on Decision and Control (CDC) (IEEE, 2016), pp. 4680-4685.

${ }^{46} \mathrm{~W}$. E. Sherwood and J. Guckenheimer, "Dissecting the phase response of a model bursting neuron,” SIAM J. Appl. Dyn. Syst. 9, 659-703 (2010).

${ }^{47} \mathrm{C}$. van Vreeswijk, "Partial synchronization in populations of pulse-coupled oscillators," Phys. Rev. E 54, 5522-5537 (1996).

${ }^{48} \mathrm{~A}$. Politi and M. Rosenblum, "Equivalence of phase-oscillator and integrateand-fire models," Phys. Rev. E 91, 042916 (2015). 